\title{
The changing landscape of oropharyngeal cancer management
}

\author{
D HAMILTON, M K KHAN, J O'HARA, V PALERI \\ ENT Department, Freeman Hospital, Newcastle upon Tyne, UK
}

\begin{abstract}
Background: Oropharyngeal cancer is increasing in prevalence in the UK and this is thought to be due to the emergence of disease related to human papilloma virus.

Method: A literature review was conducted on the diagnosis and latest management of oropharyngeal cancer.

Results: In non-smokers, human papilloma virus related disease is thought to have better outcomes, but this casts doubt on previous research which did not stratify patients according to human papilloma virus status. However, this theory provides a route for researchers to risk stratify and de-escalate treatments, and hence reduce treatment burden. In addition, the emergence of minimally invasive transoral techniques allows surgeons to remove large tumours without many of the side effects associated with radical (chemo)radiotherapy.

Conclusion: The emergence of human papilloma virus related disease and minimally invasive techniques have led the clinical and academic community to reconsider how oropharyngeal cancer is managed. Comparative and risk-stratification trials are urgently required and ongoing.
\end{abstract}

Key words: Surgical Procedures, Operative; Robotic Surgical Procedures; Laser Therapy; Oropharyngeal Neoplasms; Human Papilloma Virus

\section{Introduction}

There has been a 51 per cent increase in the incidence of oropharyngeal cancer in recent years, ${ }^{1}$ despite an overall decline in the incidence of all other head and neck cancers. ${ }^{2}$ This rise is thought to be a consequence of the increased incidence of tumours secondary to human papilloma virus (HPV) infection. The emergence of HPV-related disease has reshaped the diagnosis and investigation of this disease, and cast doubt over the effectiveness of established treatment regimens. In addition, the increasing popularity and effectiveness of modern, minimally invasive surgical techniques have changed the landscape of oropharyngeal cancer management. In this article, we discuss the current state of the art in the diagnosis and management of oropharyngeal cancer, and consider the way in which the established treatment regimens are changing.

\section{Human papilloma virus}

The emergence of HPV as a causative agent for oropharyngeal cancer was one of the most significant breakthroughs in the understanding of head and neck cancer in recent years. It led to a shift in the way in which oropharyngeal cancer is diagnosed, managed and investigated.
There has been a recent significant increase in the proportion of oropharyngeal biopsies that show evidence of HPV. ${ }^{3}$ Moreover, HPV-related oropharyngeal cancer is viewed as a distinct disease entity which has a better prognosis. ${ }^{4-6}$ However, smoking decreases survival in oropharyngeal cancer, and this effect is independent of HPV status. This means that patients with HPV-positive disease who smoke have a similar survival rate to those with HPV-negative disease. ${ }^{7}$

Sexual transmission is considered to be the primary route of HPV transmission - with an increased rate of oropharyngeal cancer in those with a history of six or more lifetime sexual partners, four or more lifetime oral sex partners or an earlier age at first sexual intercourse. ${ }^{8}$

There has been debate about a standardised test or cutoff point for reliably detecting HPV in oropharyngeal biopsies and specimens, ${ }^{9}$ with the most widely used methods being in situ hybridisation and identification of $\mathrm{p} 16$, a downstream product of HPV expression. ${ }^{10}$

The emergence of HPV-related disease has cast doubt over previous research in oropharyngeal cancer that did not stratify for HPV infection, and has caused head and neck oncologists to reconsider whether patient groups are receiving too much or too little treatment. The HPV status of the tumour does not yet affect the treatment offered to the patient. 
However, there have been large cohort studies suggesting that it should. In a study of 505 cancer patients over 8 years, patients with HPV-positive disease had a higher rate of local control and a lower rate of distant metastasis. ${ }^{11}$

It seems that if four main risk factors - smoking status, HPV status, tumour (T) stage and of nodal (N) stage - are taken into account, patients can be successfully risk stratified into low, moderate and high risk. ${ }^{6,12}$ Those in 'low-risk' groups demonstrate three-year overall survival of 93 per cent, compared to 46.3 per cent in the 'high-risk' group. ${ }^{6}$ Thus, traditionally adopted tumour-node-metastasis staging systems used for oropharyngeal cancer may be outdated in the era of HPV-related disease. In addition, well-established markers of poor prognosis in non-HPV-related disease such as extracapsular spread may not be as relevant or important in the HPV-positive group.

\section{Current management of oropharyngeal cancer}

Traditionally, available treatment options for oropharyngeal cancer were surgical resection with or without flap reconstruction, or radiotherapy with or without neck dissection. Parsons et al. combined the results from a number of published studies that compared surgery with radiotherapy. ${ }^{13}$ Following analysis of data from 51 studies with 6400 patients, the authors showed that survival and local control of oropharyngeal cancer was similar between the 2 treatment types, but the rate of severe or fatal complications was significantly higher for patients who underwent surgery. Additionally, a large, phase III randomised controlled trial, which randomised 226 patients with advanced oropharyngeal cancer to receive radiotherapy alone or radiotherapy with concomitant carboplatin and fluorouracil, showed that concomitant chemotherapy provided a survival benefit. ${ }^{14}$ Those patients receiving a combination of treatments had significantly increased overall survival ( 22 per cent vs 16 per cent) and locoregional control ( 48 per cent $v s 25$ per cent).

Following this randomised controlled trial, non-surgical management became the standard of care in the management of oropharyngeal cancer. However, over time, the number of patients suffering toxicity from the radical doses of radiotherapy to the head and neck increased: side effects were dose-dependent and worsened when combined with chemotherapy. ${ }^{15,16}$

The main short-term side effects of chemoradiotherapy include skin reactions, mucositis and infection. The long-term side effects include pharyngeal stenosis leading to dysphagia, and laryngeal scarring causing dysphonia. In the most severe cases, stenosis may lead to the requirement for tracheostomy. Severe late radiation toxicity effects include dry mouth, scarring of soft tissues and osteoradionecrosis of the mandible. ${ }^{17}$ Difficulties with teeth and oral hygiene, mouth opening, and dry mouth or sticky saliva ${ }^{15}$ may be severe and debilitating. Dysphagia is now recognised as one of the major side effects of non-surgical management. ${ }^{18}$ Many patients require supplementary enteral feeding or nutritional assistance, with a permanent gastrostomy or nasogastric tube. ${ }^{19,20}$ Dry mouth is one of the most troublesome side effects of radiotherapy, but this can be significantly reduced by employing parotid sparing plans in intensity-modulated radiotherapy. ${ }^{21}$

These complications can be common: in one of the largest trials of head and neck cancer treatment, 82 per cent of patients treated with radiotherapy and cisplatin-based chemotherapy experienced a severe toxic effect. ${ }^{22}$ Additionally, complications and severe treatment-related effects are often under-reported or under-estimated in trial reports. ${ }^{23}$

The emergence of HPV-related oropharyngeal cancer has led to question marks over the role of radical chemoradiotherapy, and the sometimes severe and unpredictable functional deficits that it creates. The difficulties experienced by patients drove cancer surgeons to develop new, minimally invasive techniques to resect oropharyngeal cancer.

\section{Transoral laser microsurgery}

Transoral laser microsurgery resection techniques were first described in $19511^{24}$ The 'lateral oropharyngectomy' was modernised and re-described by Holsinger and colleagues in $2005 .^{25}$ The first outcome analysis using this technique was published in the same year, with five-year local control rates of 89 per cent for $\mathrm{T}_{1}$ lesions, 82 per cent for $\mathrm{T}_{2}$ lesions and 63 per cent for $\mathrm{T}_{3}$ lesions. ${ }^{26}$

Steiner and colleagues were the first to use laser surgery in the treatment of the more inaccessible cancers of the tongue base, using a modification of traditional transoral instruments. ${ }^{27}$ In an investigation of 48 patients with tongue base cancer (mainly stage III and IV), Steiner's group reported an excellent local control rate of 85 per cent. Importantly, 92 per cent of patients were able to resume a normal diet in the long term following treatment. ${ }^{27}$

The role of transoral laser microsurgery in the management of oropharyngeal cancer became mainstream practice after the publication of data from 204 patients with stage III or IV oropharyngeal cancer. ${ }^{28}$ The study reported a local control rate of 97 per cent at 3 years and overall survival of 86 per cent, with 87 per cent of patients having normal swallowing.

The functional results after the use of transoral laser microsurgery in oropharyngeal cancer are also encouraging. In one of the first preliminary reports of a comparison between chemoradiotherapy and transoral laser microsurgery in the treatment of advanced stage oropharyngeal cancer, those treated with chemoradiotherapy were found to have a greater deterioration in swallowing function at three months compared to those treated with transoral laser microsurgery, when assessed using the MD Anderson Dysphagia Inventory, the Normalcy of Diet subsection of the Performance Status Scale and a timed water swallow test. ${ }^{29}$ 


\section{Transoral robotic surgery}

Transoral robotic surgery provides the operating surgeon with some important benefits over transoral laser microsurgery, the most striking of which is that it allows the surgeon to use two hands to manipulate and resect the tumour. The operator virtually controls two instruments from across the operating theatre; indeed the robot allows hand movements in seven degrees of freedom (a range of planes which would be impossible with the human wrist), with the elimination of tremor and 75 per cent higher resolution.

The use of transoral robotic surgery in the head and neck was pioneered and developed first in airway mannequins, ${ }^{30}$ followed by canine subjects ${ }^{31}$ and cadavers, and then eventually procedures were performed in human participants on tumours in the tongue base ${ }^{32}$ and tonsil. ${ }^{33}$ Since its development, transoral robotic surgery has become more prevalent as more departments have invested in the necessary technology. Initial reports of outcomes have been favourable. One small cohort study (89 patients) demonstrated two-year survival rates of 86.5 per cent. ${ }^{34}$ Another study (177 patients) reported no intra-operative or immediate post-operative mortality, a positive margin rate of 4.3 per cent, a long-term tracheostomy rate of 2.3 per cent, and a long-term gastrostomy tube rate of 5 per cent. ${ }^{35}$

\section{Surgical trials}

Although great strides have been made in the modernisation of oropharyngeal cancer treatment, initial promising results should be treated with relative caution. The historical move from surgery to radiotherapy was based on well-conducted, large randomised trials, whereas the evidence on which transoral laser microsurgery and transoral robotic surgery is based relies heavily on smaller case series, and there is a current lack of well-conducted, comparative studies. For this reason, researchers have recently set up large, randomised studies with two main aims: to stratify the intensity of treatment according to patients' likelihood of survival, and to compare new minimally invasive surgical techniques with radiotherapy (with or without chemotherapy).

\section{Risk-stratification trials}

As previously discussed, HPV-positive oropharyngeal cancer is associated with a higher chance of survival than HPV-negative disease. The predictive power of HPV status can be increased if it is combined with other clinical characteristics of the disease. Currently, the HPV status of the patient does not have an effect on the treatment recommended, but this HPV-positive patient group could potentially have their treatment de-intensified.

The 'De-ESCALaTE' (Determination of Epidermal growth factor receptor inhibitor (cetuximab) versus Standard Chemotherapy (cisplatin) early And Late Toxicity Events in Human Papillomavirus positive oropharyngeal squamous cell carcinoma) trial and the Radiation Therapy Oncology Group's 'RTOG 1016' trial are testing this hypothesis directly by randomising patients with stage III and stage IV oropharyngeal cancer to receive radiotherapy with either concurrent cetuximab or concurrent cisplatin. Their primary outcomes are different, however. The De-ESCALaTE trial has a primary outcome of toxicity reactions, and has secondary outcomes of swallow function and survival. In contrast, the RTOG 1016 measures overall survival and quality of life. These trials aim to answer the simple primary question: does the HPV status of the tumour allow the oncologist to change a more toxic drug (cisplatin) for one with a less severe side effect profile (cetuximab) without compromising survival?

Surgical resection provides another potential route of treatment de-intensification. It can provide more detailed information about tumour size, extent, spread and stage, and a large tumour specimen on which histological tumour grade and the presence of any nodal metastases can be determined. A neck dissection also provides reliable information about the presence of extracapsular spread, a histological finding which usually denotes a poorer prognosis requiring postoperative chemoradiotherapy. ${ }^{36}$ However, because of the positive effect of HPV status on survival, many theorise that in HPV-positive disease, extracapsular spread of nodal metastases should not automatically mean that patients require the addition of cisplatin to their postoperative radiotherapy.

This is the premise of the 'ADEPT' (Adjuvant Deescalation Extracapsular Spread, p16+ Transoral) trial, in which patients undergo primary transoral tumour resection and neck dissection. Those with HPV-positive disease and clear margins, but with extracapsular spread are then randomised to receive either radiotherapy or radiotherapy and cisplatin. Progression-free survival and locoregional recurrence are the primary outcome measures.

In the 'PATHOS' (Post-operative adjuvant treatment for HPV-positive tumours) trial, attempts are made to risk stratify patients further. Specifically, patients undergo transoral resection of an HPV positive oropharyngeal cancer and are subsequently categorised as low risk (no adverse histological features), moderate risk (stage III tumours, $\mathrm{N}_{2 \mathrm{a}-\mathrm{b}}$, with adverse histological characteristics or close margins) or high risk (positive margins or extracapsular spread). Those with low risk are given no adjuvant post-operative treatment, and those with moderate or high risk are randomised to receive various doses of radiotherapy with or without the addition of cisplatin.

The Eastern Co-operative Oncology Group's 'ECOG 3311' trial has a similar premise: tumours are resected transorally and neck dissection is performed. Similarly, this study includes only patients with HPV-positive disease, and risk stratifies them based on tumour stage, nodal metastases, extracapsular spread and peri-neural invasion. Low-risk patients 
$\left(\mathrm{T}_{1-2}, \mathrm{~N}_{0-1}\right.$, clear margins, with no extracapsular spread) are given no further treatment, and high-risk patients (positive margins, extensive extracapsular spread or more than five lymph nodes) are given post-operative chemoradiotherapy. However, those with intermediate risk (close margins, minimal extracapsular spread, $\mathrm{N}_{1-2 \mathrm{~b}}$ neck disease or peri-neural invasion) are randomised to receive high- or low-dose radiotherapy.

Although de-intensification of treatment in those with HPV-positive, low-risk disease is a popular current research question, it should also be considered whether those with higher-risk disease (HPV-negative, extensive neck disease) are receiving adequately radical doses of therapy. Indeed, as traditionally researchers did not stratify patients on the basis of HPV status, it is possible that the current standard of concomitant chemoradiotherapy is also not an aggressive enough option for those with higher-risk HPV-negative disease.

The 'CompARE' (Phase III randomised controlled trial Comparing Alternative Regimens for escalating treatment of intermediate and high-risk oropharyngeal cancer) trial aims to address this subject by randomising those deemed to be of intermediate risk (HPV-positive oropharyngeal cancer with $\mathrm{N}_{2 \mathrm{~b}+}$ disease and greater than 10 pack-year smoking history) or high risk (HPVnegative, with extracapsular spread) to receive combinations of: concomitant chemoradiotherapy, surgery and concomitant chemoradiotherapy, induction chemotherapy then concomitant chemoradiotherapy, or doseescalated chemoradiotherapy. The outcome measure in that trial is overall survival.

\section{Comparison trials}

Although the majority of trials in oropharyngeal cancer investigate risk stratification, a smaller number instead compare newer minimally invasive surgical techniques directly with the current standard of care.

In the 'ORATOR' (Phase II Randomized Trial for Early-stage Squamous Cell Carcinoma of the Oropharynx: Radiotherapy vs Trans-oral Robotic Surgery) trial, patients with early-stage oropharyngeal cancer $\left(\mathrm{T}_{1-2}\right.$, with less than two neck nodes) are randomised to receive radiotherapy or transoral robotic resection with neck dissection. The primary outcome measure in that trial is quality of life.

The Radiation Therapy Oncology Group's 'RTOG 1221 ' trial is performing a similar comparison in highrisk advanced disease patients. Stage III and IV, HPVnegative oropharyngeal cancer patients are randomised to receive concurrent cisplatin and radiotherapy, or transoral surgery and neck dissection. Those patients who undergo surgical resection then receive adjuvant therapy based on the risk profile post-resection. The primary outcome measure in that trial is progression-free survival.

Current research is focused on modernising the risk stratification and treatment of patients with oropharyngeal cancer. Indeed, patients with HPV-positive or HPV-negative oropharyngeal cancer have a large selection of recruiting trials for which they are eligible. Once available, the outcomes of this research should allow the most pressing current management questions to be answered. These questions include: can those with low-risk disease have their treatment de-intensified? Should those with high-risk disease have their treatment intensified? Can surgery provide a route for this de-intensification? Is surgery a better, safer option for some patients?

\section{Conclusion}

Oropharyngeal cancer management has presented the head and neck cancer clinical and academic community with a formidable challenge. Non-surgical treatment regimens, in the form of radiotherapy with concomitant cisplatin, remain the standard of care. However, a combination of poor functional outcomes in these patients, coupled with the emergence and popularisation of novel, minimally invasive surgical techniques, are challenging this dogma. Excellent tumour control and functional outcomes in selected patients treated with transoral laser microsurgery or transoral robotic surgery have meant that the professional community treating these tumours has had to reconsider the role of surgery in the treatment of head and neck cancer.

The increasing incidence of oropharyngeal cancer has led to it being one of the most common presentations to the head and neck cancer clinic. The emergence of HPVrelated disease has called into question current established thinking about the best way to diagnose and manage oropharyngeal cancer. Well-designed, randomised clinical trials are central to the progression and modernisation of care in oropharyngeal cancer, and new patients should be given the opportunity to enrol in them. Almost every patient presenting with oropharyngeal cancer will be eligible for inclusion into one of the trials discussed above, and the current uncertainty regarding the standard of care means that eligible patients should, when possible, receive treatment within a trial setting. However, although the results of such trials will provide clinicians and patients with reliable data to base treatment decisions on, they will not eliminate treatment choice. Oropharyngeal cancer patients will still face a complex disease with a range of treatment options that vary greatly in terms of their delivery and outcome. Although trials are essential to assess the effectiveness and safety of transoral laser microsurgery and transoral robotic surgery, the choice of treatment will still require the clinician and patient to balance treatment outcomes, risks and complications with their own values and priorities. Indeed, the modernisation of oropharyngeal cancer management will force clinicians to examine individual patient and tumour characteristics when making treatment decisions.

\footnotetext{
References

1 Cancer Research UK. Oral cancer incidence statistics, 2013. In: http://info.cancerresearchuk.org/cancerstats/types/oral/incidence [27 September 2016]
} 
2 National Cancer Institute. Surveillance, Epidemiology and End Results Program, 2013. In: http://seer.cancer.gov/ [27 September 2016]

3 Nasman A, Attner P, Hammarstedt L, Du J, Eriksson M, Giraud $\mathrm{G}$ et al. Incidence of human papillomavirus (HPV) positive tonsillar carcinoma in Stockholm, Sweden: an epidemic of viralinduced carcinoma? Int J Cancer 2009;125:362-6

4 Fakhry C, Westra WH, Li S, Cmelak A, Ridge JA, Pinto H et al. Improved survival of patients with human papillomavirus-positive head and neck squamous cell carcinoma in a prospective clinical trial. J Natl Cancer Inst 2008;100:261-9

5 Worden FP, Kumar B, Lee JS, Wolf GT, Cordell KG, Taylor JM et al. Chemoselection as a strategy for organ preservation in advanced oropharynx cancer: response and survival positively associated with HPV16 copy number. J Clin Oncol 2008;26: $3138-46$

6 Ang KK, Harris J, Wheeler R, Weber R, Rosenthal DI, NguyenTan PF et al. Human papillomavirus and survival of patients with oropharyngeal cancer. $N$ Engl J Med 2010;363:24-35

7 Gillison ML, Zhang Q, Jordan R, Xiao W, Westra WH, Trotti A et al. Tobacco smoking and increased risk of death and progression for patients with p16-positive and p16-negative oropharyngeal cancer. J Clin Oncol 2012;30:2102-11

8 Heck JE, Berthiller J, Vaccarella S, Winn DM, Smith EM, Shan'gina $\mathrm{O}$ et al. Sexual behaviours and the risk of head and neck cancers: a pooled analysis in the International Head and Neck Cancer Epidemiology (INHANCE) consortium. Int $J$ Epidemiol 2010;39:166-81

9 Mehanna H, Jones TM, Gregoire V, Ang KK. Oropharyngeal carcinoma related to human papillomavirus. BMJ 2010;340: c1439

10 Singhi AD, Westra WH. Comparison of human papillomavirus in situ hybridization and p16 immunohistochemistry in the detection of human papillomavirus-associated head and neck cancer based on a prospective clinical experience. Cancer 2010;116:2166-73

11 O'Sullivan B, Huang SH, Siu LL, Waldron J, Zhao H, PerezOrdonez B et al. Deintensification candidate subgroups in human papillomavirus-related oropharyngeal cancer according to minimal risk of distant metastasis. J Clin Oncol 2013;31: $543-50$

12 Granata R, Miceli R, Orlandi E, Perrone F, Cortelazzi B, Franceschini $\mathrm{M}$ et al. Tumor stage, human papillomavirus and smoking status affect the survival of patients with oropharyngeal cancer: an Italian validation study. Ann Oncol 2012;23:1832-7

13 Parsons JT, Mendenhall WM, Stringer SP, Amdur RJ, Hinerman RW, Villaret DB et al. Squamous cell carcinoma of the oropharynx: surgery, radiation therapy, or both. Cancer 2002;94:2967-80

14 Denis F, Garaud P, Bardet E, Alfonsi M, Sire C, Germain T et al. Final results of the 94-01 French Head and Neck Oncology and Radiotherapy Group randomized trial comparing radiotherapy alone with concomitant radiochemotherapy in advanced-stage oropharynx carcinoma. J Clin Oncol 2004;22:69-76

15 Abendstein H, Nordgren M, Boysen M, Jannert M, Silander E, Ahlner-Elmqvist $\mathrm{M}$ et al. Quality of life and head and neck cancer: a 5 year prospective study. Laryngoscope 2005;115: 2183-92

16 Rogers SN, Ahad SA, Murphy AP. A structured review and theme analysis of papers published on 'quality of life' in head and neck cancer: 2000-2005. Oral Oncol 2007;43:843-68

17 Denis F, Garaud P, Bardet E, Alfonsi M, Sire C, Germain T et al. Late toxicity results of the GORTEC 94-01 randomized trial comparing radiotherapy with concomitant radiochemotherapy for advanced-stage oropharynx carcinoma: comparison of LENT/SOMA, RTOG/EORTC, and NCI-CTC scoring systems. Int J Radiat Oncol Biol Phys 2003;55:93-8

18 Wilson JA, Carding PN, Patterson JM. Dysphagia after nonsurgical head and neck cancer treatment: patients' perspectives. Otolaryngol Head Neck Surg 2011;145:767-71

19 Paleri V, Patterson J. Use of gastrostomy in head and neck cancer: a systematic review to identify areas for future research. Clin Otolaryngol 2010;35:177-89

20 Moor JW, Patterson J, Kelly C, Paleri V. Prophylactic gastrostomy before chemoradiation in advanced head and neck cancer: a multiprofessional web-based survey to identify current practice and to analyse decision making. Clin Oncol ( $R$ Coll Radiol) 2010;22:192-8
21 Nutting CM, Morden JP, Harrington KJ, Urbano TG, Bhide SA, Clark $\mathrm{C}$ et al. Parotid-sparing intensity modulated versus conventional radiotherapy in head and neck cancer (PARSPORT): a phase 3 multicentre randomised controlled trial. Lancet Oncol 2011;12:127-36

22 Forastiere AA, Goepfert H, Maor M, Pajak TF, Weber R, Morrison $\mathrm{W}$ et al. Concurrent chemotherapy and radiotherapy for organ preservation in advanced laryngeal cancer. $N$ Engl $J$ Med 2003;349:2091-8

23 Trotti A, Pajak TF, Gwede CK, Paulus R, Cooper J, Forastiere A et al. TAME: development of a new method for summarising adverse events of cancer treatment by the Radiation Therapy Oncology Group. Lancet Oncol 2007;8:613-24

24 Huet PC. Electrocoagulation in epitheliomas of the tonsils. Ann Otolaryngol 1951;68:433-42

25 Holsinger FC, McWhorter AJ, Ménard M, Garcia D, Laccourreye O. Transoral lateral oropharyngectomy for squamous cell carcinoma of the tonsillar region: I. Technique, complications, and functional results. Arch Otolaryngol Head Neck Surg 2005;131:583-91

26 Laccourreye O, Hans S, Ménard M, Garcia D, Brasnu D, Holsinger FC. Transoral lateral oropharyngectomy for squamous cell carcinoma of the tonsillar region: II. An analysis of the incidence, related variables, and consequences of local recurrence. Arch Otolaryngol Head Neck Surg 2005;131:592-9.

27 Steiner W, Fierek O, Ambrosch P, Hommerich CP, Kron M. Transoral laser microsurgery for squamous cell carcinoma of the base of the tongue. Arch Otolaryngol Head Neck Surg 2003;129:36-43

28 Haughey BH, Hinni ML, Salassa JR, Hayden RE, Grant DG, Rich JT et al. Transoral laser microsurgery as primary treatment for advanced-stage oropharyngeal cancer: a United States multicenter study. Head Neck 2011;33:1683-94

29 O'Hara J, Cosway B, Muirhead C, Leonard N, Goff D, Patterson J. Transoral laser microsurgery \pm adjuvant therapy versus chemoradiotherapy for stage III and IVA oropharyngeal squamous cell cancer: preliminary comparison of early swallowing outcomes. Head Neck 2015;37:1488-94

30 Hockstein NG, Nolan JP, O’Malley BW Jr, Woo YJ. Robotic microlaryngeal surgery: a technical feasibility study using the daVinci surgical robot and an airway mannequin. Laryngoscope 2005;115:780-5

31 O’Malley BW Jr, Weinstein GS, Hockstein NG. Transoral robotic surgery (TORS): glottic microsurgery in a canine model. $J$ Voice 2006;20:263-8

32 O'Malley BW Jr, Weinstein GS, Snyder W, Hockstein NG. Transoral robotic surgery (TORS) for base of tongue neoplasms. Laryngoscope 2006;116:1465-72

33 Weinstein GS, O'Malley BW Jr, Snyder W, Sherman E, Quon H. Transoral robotic surgery: radical tonsillectomy. Arch Otolaryngol Head Neck Surg 2007;133:1220-6

34 White HN, Moore EJ, Rosenthal EL, Carroll WR, Olsen KD, Desmond RA et al. Transoral robotic-assisted surgery for head and neck squamous cell carcinoma: one- and 2-year survival analysis. Arch Otolaryngol Head Neck Surg 2010;136:1248-52

35 Weinstein GS, O’Malley BW Jr, Magnuson JS, Carroll WR, Olsen KD, Daio L et al. Transoral robotic surgery: a multicenter study to assess feasibility, safety, and surgical margins. Laryngoscope 2012;122:1701-7

36 Cooper JS, Pajak TF, Forastiere AA, Jacobs J, Campbell BH, Saxman SB et al. Postoperative concurrent radiotherapy and chemotherapy for high-risk squamous-cell carcinoma of the head and neck. $N$ Engl J Med 2004;350:1937-44

Address for correspondence:

Mr David Hamilton,

ENT Department,

Freeman Hospital,

High Heaton,

Newcastle upon Tyne NE7 7DN, UK

E-mail: david.hamilton@ncl.ac.uk

Mr D Hamilton takes responsibility for the integrity of the content of the paper

Competing interests: None declared 